\title{
Prostate Cancer: How Young is too Young?
}

\author{
Sahil Gupta ${ }^{a} \quad$ Arjun Gupta $^{\mathrm{b}} \quad$ Ashish K. Saini $^{\mathrm{a}} \quad$ Kaustav Majumder $^{\mathrm{c}}$ \\ Kalpana Sinha ${ }^{a} \quad$ Anurag Chahal $^{\mathrm{a}}$
}

aAll India Institute of Medical Sciences, New Delhi, India; 'bUT Southwestern Medical Center, Dallas, Tex.; 'University of Minnesota

Medical Center, Minneapolis, M.N., USA

\section{Key Words}

Prostate • Prostate cancer • Urology

\begin{abstract}
Prostate cancer is the most common non-cutaneous malignancy in men. It is generally considered a cancer of the elderly, and the median age of presentation is 68 years. However $10 \%$ of new diagnoses in the USA occur in men aged $\leq 55$ years. This may be due to more prevalent screening nowadays, and may also reflect the diagnosis of an increasingly recognized but underappreciated entity, i.e. early-onset prostate cancer. Patients with early onset prostate cancer pose unique challenges. Current data suggest that early-onset prostate cancer is a distinct phenotype-from both an etiological and clinical perspective - that deserves further attention. We present a case of a 28 -year-old man who presented with lower urinary tract symptoms and was diagnosed with advanced stage prostate cancer.
\end{abstract}

Copyright $\odot 2015$ S. Karger AG, Basel

\section{Background}

Prostate cancer is most common malignancy in men, with presentation in elderly males. Recent studies show increase in incidence of prostate cancer in young males. Prostate cancer in younger age group is generally undifferentiated and associated with poor prognosis. Our case represents one of the youngest reported cases of prostate cancer.

Our case demonstrates that prostate cancer is not only "disease of old age", as believed earlier, and should be kept as differential when treating young males for lower urinary tract symptoms.

\section{Case Report}

A 28-year-old man presented with lower abdominal pain, weak urine stream, nocturia and urinary hesitancy for 3 weeks. Symptoms had gradually become worse to the point where he could no longer void without straining and had 1 episode of acute urinary retention. He also complained of lower back ache and pressure for the past 4 days. He denied fever and other constitutional symptoms. He did not endorse burning during micturition, changes in bowel habitus, erectile dysfunction or painful erection and had not noticed any blood in urine or semen. No history of urinary tract infection, nephrolithiasis, malignancy or neurological disease was reported. The patient had no known medical conditions.

On exam, he was afebrile with pulse rate $82 / \mathrm{min}$, blood pressure $128 / 76 \mathrm{mmHg}$, respiratory rate $16 / \mathrm{min}$. General physical and systemic examination was unremarkable. Digital rectal exam revealed a hard, non-tender, multinodular prostate with irregular surface.

\section{KARGER}

Fax +4161306 1234

E-Mail karger@karger.ch

www.karger.com
(C) 2015 S. Karger AG, Basel

1015-9770/14/0093-0212\$38.00/0

Accessible online at:

www.karger.com/cur
Sahil Gupta

All India Institute of Medical Sciences,

Hostel No. 8, Room No. 2

Boys Hostel, AIIMS Campus

New Delhi (India)

E-Mail sahil.self@gmail.com 


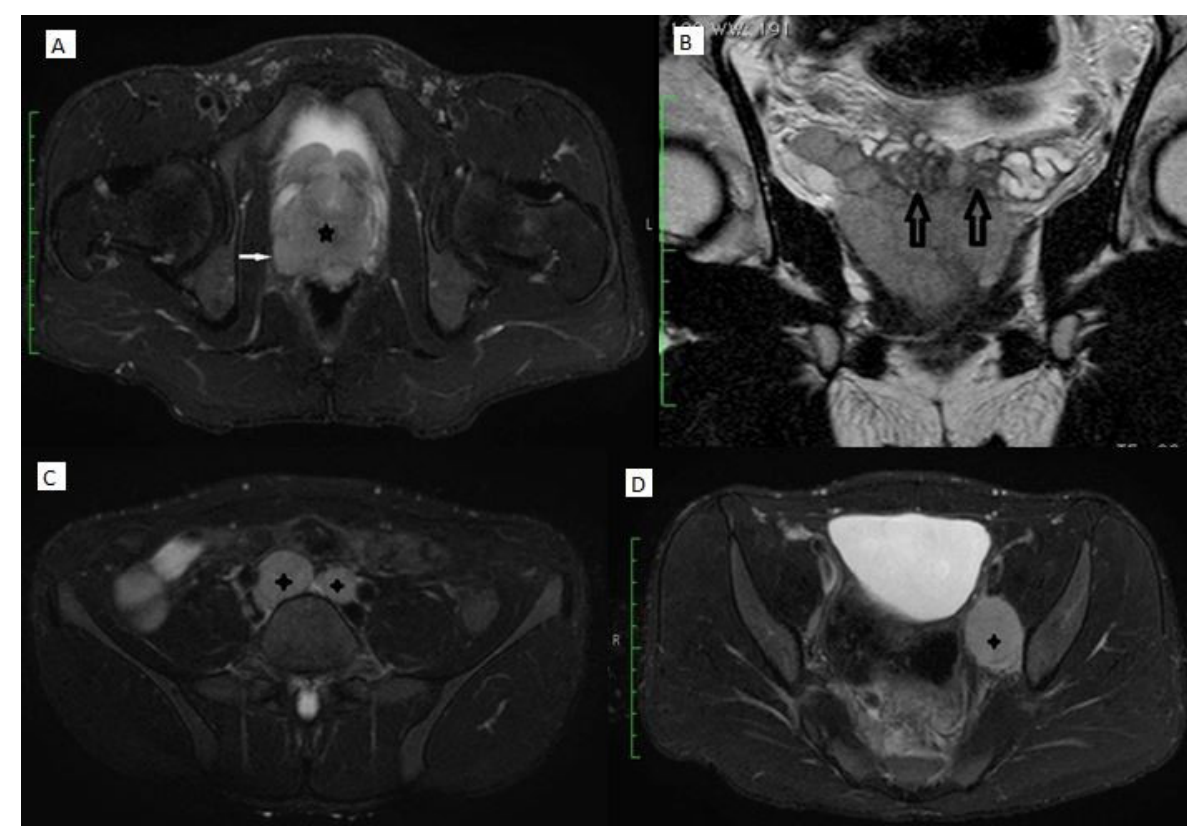

Fig. 1. Radiographic findings (MRI). A Large T2 hypo intense mass (*) almost replacing the prostate gland with extra capsular invasion (arrow); B T2 coronal images show invasion of mass into bilateral seminal vesicles (arrows); C Enlarged bilateral common iliac lymph nodes (+); D Enlarged left internal iliac lymph node (+).

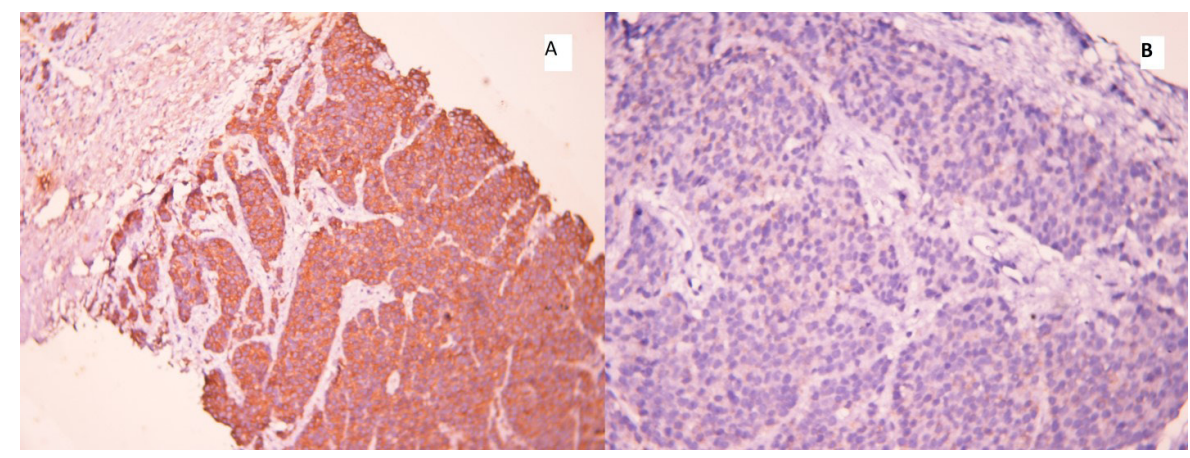

Fig. 2. Histopathological features. Immunohistochemistry showing strong pan-cytokeratin (A) and AMACR (B) positivity.

\section{Investigations}

Laboratory analysis revealed white blood cell count $8.3 \times 10^{9} / 1$, hemoglobin $13.2 \mathrm{~g} / \mathrm{dl}$, platelet count $214 \times$ $10^{9} / 1$. Urinalysis, urine culture, electrolytes including fasting and post prandial blood sugar, serum calcium and alkaline phosphatase were unremarkable. Serum creatinine was $0.8 \mathrm{mg} / \mathrm{dl}$ and prostate specific antigen (PSA) level was $5.85 \mathrm{ng} / \mathrm{ml}$ (normal PSA 0-4 ng/ml).
Pelvic ultrasonography demonstrated a heterogeneous prostatic mass with enlarged aortocaval and left internal iliac lymph nodes each. Magnetic resonance imaging of the abdomen and pelvis revealed an enlarged multi-lobulated prostate with marked diffusion restriction, and extra capsular extension into the bilateral seminal vesicles and the bladder neck (fig. 1). There were also multiple enlarged pelvic lymph nodes. Abdominopelvic viscera appeared normal. 
A transrectal ultrasound guided biopsy of the prostate demonstrated adenocarcinoma of prostate with Gleason score $4+5$. Immunohistochemistry confirmed the diagnosis of poorly differentiated adenocarcinoma of prostate with sections showing focal PSA positivity along with strong pan-cytokeratin, AMACR and synaptophysin positivity (fig. 2). Ki 67 index was elevated to $40 \%$. Sections were negative for CK 7, CK 20, TTF1, MIC 2 and myogenin. The patient refused genetic testing.

\section{Differential Diagnosis}

The patient's presentation: lower abdominal pain, weak urinary stream and nocturia; points towards urinary tract infection which was ruled out by unremarkable urine analysis and sterile urine culture report. Another diagnosis which comes to mind is diabetes mellitus which was ruled out by normal fasting and post prandial blood sugar levels.

Nephrolithiasis is a very important differential as the patient presents with lower urinary tract symptoms and if the calculus is in the bladder or the urethra, it can cause poor urinary stream. Our patient's urinary and serum calcium and phosphate levels were unremarkable and pelvic ultrasonography showed no hydroureteronephrosis or calculus in ureters, bladder or urethra.

With a history of lower urinary tract symptoms, urethral stricture is also a possibility but there was no history of any trauma or previous catheterization. This makes traumatic stricture an unlikely diagnosis. No recurrent or previous episodes of urinary tract infections or burning micturition rules out urethral stricture as a diagnosis.

Prostatitis can present as urinary tract infection, but on digital rectal examination prostate was non-tender and also urine analysis was unremarkable and had no leukocytes or any microorganism. Also transrectal ultrasonography didn't reveal any signs of prostatitis.

Digital rectal examination revealed nodular hard prostate which raised the suspicion of prostate cancer which was then confirmed by transrectal ultrasonography and biopsy.

\section{Treatment}

In view of the extra capsular extension and lymph node spread, androgen deprivation therapy (ADT) was offered to the patient who chose bilateral orchidectomy over chemical/hormonal androgen deprivation. Semen preservation was performed and bilateral orchidectomy performed without incident. He received daily adjuvant hormonal chemotherapy with bicalutamide after surgery.

\section{Outcome and Follow-up}

Follow-up at 12-months after presentation showed no urological complaints, with a PSA level of $0.45 \mathrm{ng} / \mathrm{ml}$. $\mathrm{He}$ is continuing to receive bicalutamide treatment.

\section{Discussion}

To the best of our knowledge, the literature on prostate cancer contains $<30$ reported cases of prostate cancer among men $\leq 40$ years of age, with an incidence of $0.8-1.1 \%[1-5]$.

PSA-based screening has induced an important age migration effect in which the incidence of prostate cancer has increased in men of lower age group.

Early onset prostate cancer (diagnosed in men $<55$ years of age) is considered a different clinical entity from prostate cancer diagnosed at an older age. A number of large population based studies have demonstrated poor survival among patients $<50$ years of age with advanced prostate cancer or unknown stage disease as compared to older patients [6-13]. PSA level is lower in these patients due to poorly differentiated adenocarcinoma of prostate. So in poorly differentiated carcinoma, PSA level is not indicative of prostate cancer.

Patients diagnosed with high grade tumors (Gleason score of 8-10) at ages 35-44 are also at higher odds of succumbing to prostate cancer as compared to patients aged 65-74 years. Moreover a strong genetic component has been associated with early onset prostate cancer.

Lange et al. [14] reported that men with early onset prostate cancer are more likely to have a greater number of genetic variants, which are associated with an increased risk of prostate cancer, as compared to older patients. Although a majority of patients with early onset prostate cancer are diagnosed with moderately differentiated disease, the management of patients with early onset prostate cancer poses unique clinical challenges.

Cases with organ limited prostate cancer may benefit from prostatectomy but same is not the case in locally advanced or metastatic prostate cancer. Patients who are diagnosed with prostate cancer with associated lymph node involvement are broadly categorized into 2 subgroups: patients who have detectable lymph nodes on imaging 
$(\mathrm{dN}+)$ and patients who have no detectable lymph node involvement on imaging but have node positive disease at time of surgery $(\mathrm{pN}+)$. According to the 2013 National

Comprehensive Cancer Network guidelines [15], patients with $\mathrm{dN}+$ disease should be managed with ADT or RT-ADT.

On the other hand, patients with lymph node involvement detected at radical prostatectomy are offered observation, ADT or RT-ADT. ADT can be achieved medically by hormonal drugs or surgically by orchidectomy. Our patient had locally advanced disease with imaging identifying local LN spread and surgery was not conducted. He was appropriately managed with ADT as per his wishes.

\section{Learning Points/Take Home Messages}

1. With changing environmental factors and PSA screening, a larger number and proportion of young males with prostate cancer are coming into attention.

2. Most young patients with prostate cancer have moderately differentiated, organ confined disease.

3 . Given the otherwise longer life expectancy in younger patients, treatment should be initiated promptly rather than using the wait and watch method generally used in older age group males.

4. There is more risk of treatment related adverse effects in the younger population for the same reason.

\section{References}

1 Yamamoto S, Senzaki A, Yamagiwa K, Tanaka T, Oda T: Prostatic carcinoma in a young adult: a case report. Hinyokika Kiyo 1990;36:617-622.

2 Roberts JT, Essenhigh DM: Adenocarcinoma of prostate in 40-year-old body-builder.Lancet 1986;2(8509):742.

3 Davis BE, Weigel JW: Adenocarcinoma of the prostate discovered in 2 young patients following total prostatovesiculectomy for refractory prostatitis. J Urol 1990;144:744745.

4 Ruska KM, Partin AW, Epstein JI, Kahane $\mathrm{H}$ : Adenocarcinoma of the prostate in men younger than 40 years of age: diagnosis and treatment with emphasis on radical prostatectomy findings. Urology 1999;53:1179-1183.

5 D'Aprile M, Santini D, Di Cosimo S, Gravante G, Vincenzi B, Spoto S, Costantino S, Rabitti C, Tonini G: Atypical case of metastatic undifferentiated prostate carcinoma in a 36 years old man: clinical report and literature review. Clin Ter 2000;151:371-374.
6 Gronberg H, Damber JE, Jonsson H, Lenner $P$ : Patient age as a prognostic factor in prostate cancer. J Urol 1994;152:892-895.

7 Harrison GS: The prognosis of prostatic cancer in the younger man. $\mathrm{Br} \mathrm{J}$ Urol 1983;55:315-320.

8 Huben R, Natarajan N, Pontes E, Mettlin C, Smart CR, Murphy GP: Carcinoma of prostate in men less than fifty years old. Data from American College of Surgeons' National Survey. Urology 1982;20:585-588.

9 Johnson DE, Lanieri JP Jr, Ayala AG: Prostatic adenocarcinoma occurring in men under 50 years of age. J Surg Oncol 1972;4:207216.

10 Lin DW, Porter M, Montgomery B: Treatment and survival outcomes in young men diagnosed with prostate cancer: a population-based cohort study. Cancer 2009;115: 2863-2871.
11 Merrill RM, Bird JS: Effect of young age on prostate cancer survival: a population based assessment (United States). Cancer Causes Control 2002;13:435-443.

12 Tjaden HB, Culp DA, Flocks RH: Clinical adenocarcinoma of the prostate in patients under 50 years of age. J Urol 1965;93:618 621

13 Wilson JM, Kemp IW, Stein GJ: Cancer of the prostate. Do younger men have a poorer survival rate? Br J Urol 1984;56:391-396.

14 Lange EM, Salinas CA, Zuhlke KA, Ray AM, Wang Y, Lu Y, Ho LA, Luo J, Cooney KA: Early onset prostate cancer has a significant genetic component. Prostate 2012;72: 147-156.

15 Mitin T, Blute M, Lee R, Efstathiou J: Management of lymph node-positive prostate cancer: the role of surgery and radiation therapy. Oncology (Williston Park) 2013;27:647-655 